\title{
Screening, evaluation of vibriocidal activity and characterization of bioactive substance from marine cyanobacteria
}

\author{
Chandru $\mathbf{S}^{1 *}$, Jenitta Hephzibah $\mathrm{A}^{\mathbf{2}}$, Rajesh $\mathrm{P}^{1}$ and Rajesh Kannan $\mathrm{V}^{1}$ \\ ${ }^{1}$ Department of Microbiology, Bharathidasan University, Tiruchirappalli-620024.Tamil Nadu, India. \\ ${ }^{2}$ Department of Microbiology, Urumu Dhanalakshmi College, Tiruchirappalli-620019, Tamil Nadu, India.
}

Accepted 21 February, 2013

\begin{abstract}
The pathogens of Vibrio sp. mainly cause three diseases such as gastroenteritis, wound infection and septicaemia; they can also cause food borne infection. At present, marine cyanobacterial species such as Oscillatoria sp. and Lyngbya sp. were extracted using aqueous, ethyl acetate and pyridine extracts. The marine cyanobacterial extracts were evaluated for antivibrio activity by agar well diffusion method against Vibrio sp., such as Vibrio alginolyticus, Vibrio cholera and Vibrio fluvialis. Pyridine extract of Oscillatoria sp. shows maximum zone of inhibition and the crude extract was further separated by thin layer chromatography; and the separated fraction was evaluated for vibriocidal activity. The activated fraction was further analyzed by Fourier transforms infrared spectroscopy (FTIR) and the fraction showed the presence of phenols, aminoacids, sterols and saponins. The present study concludes that, the antivibrio metabolites of marine cyanobacteria are of special interest in the development of new harmless environment.
\end{abstract}

Key words: Vibrio sp., marine cyanobacteria, vibriocidal compound, TLC, FTIR.

\section{INTRODUCTION}

This increase in antibiotic resistant bacteria is a serious issue because of the constant concern of reduced efficiency of antibiotics in the treatment of human diseases. In turn, this has given rise to research on new methods of disease prevention in which many researchers have opted to use probiotics as they are environmentally safe and cost effective (Gómez-Gil et al., 2000;Verschuere et al., 2000; Vine, 2004). Cyanobacteria are rich sources of vitamins, essential amino acids, minerals and fatty acids, as well as carotenoid pigments (Takeuchi et al., 2002). They have contributed to the discovery of numerous secondary metabolites as sources of new pharmaceuticals and biotechnological products with a broad array of chemical structures; some of these may be responsible for influencing the microbiota of their environment (Marshall et al., 2005; Jaiswal et al., 2008).

Cyanobacteria from local habitats seem to be a source of new potential active substances that could contribute to reduction of the number of bacteria, fungi, viruses and other microorganisms (Mundt et al., 2001). Isolation of bioactive compounds from cyanobacteria is done with two objectives: one is to discover new compounds for pharmaceutical, agricultural or biological application; the other is for the better understanding of the interactions of individual organisms within their natural communities. For each of these purposes, there is a need to screen new organisms (Rania and Hala, 2008). Pathogenic Vibrio can cause foodborne illness (infection), usually associated with 
eating undercooked seafood. Vibrio infections are largely classified into two distinct groups: Vibrio cholera infections and non-cholera Vibrio infections. Historically, the non-cholera Vibrio species are classified as halophilic or non-halophilic, depending on their requirement of sodium chloride for growth (Michael Hogan 2010).

\section{MATERIALS AND METHODS}

\section{Collection of marine cyanobacterial samples}

Cyanobacterial culture was obtained from Microbial Germ Plasm Culture Collection Unit (MGPCCU), Sri Gowri Biotech Research Academy at Thanjavur and used for present investigation. The cynaobacterial morphotypes such as filamentous nature, size, shape of vegetative cells and akinetes were identified and photographed under Nikon digital microscope. Identification of the cyanobcterial isolates were carried out by using the taxonomic publication of Desikachary (1959) (Figure 1).

\section{Preparation of solvent extracts from marine cyanobacteria}

$50 \mathrm{mg}$ of both marine cyanobacterial samples was extracted with three different solvent systems- $3 \mathrm{ml}$ of pyridine, ethyl acetate and aqueous extracts in a beaker for $24 \mathrm{~h}$ at room temperature. Then the solvent suspension was centrifuged at $700 \times g$ for $6 \mathrm{~min}$. The supernatant was drawn with a pipette and $100 \mu \mathrm{L}$ of the aqueous extract was transferred to each well, and air-dried before using. The pre-weighed marine cyanobacterial cultures were crushed using mortar and pestle. The samples were mixed and then left with the extraction fluid for $10 \mathrm{~min}$. The samples were centrifuged at $700 \times \mathrm{g}$ for $6 \mathrm{~min}$ and the supernatant was transferred to a clean $2 \mathrm{~mL}$ eppendorf tube. The solvent was evaporated to dryness (Perez et al., 1990).

\section{Screening of antivibrio activity of marine cyanobacteria}

The antivibrio activity of marine cyanobacterial extracts was studied by agar-well diffusion methods, using nutrient agar (NA). The tested vibrio cultures were evenly spread over the appropriate media (NA) by using a sterile cotton swab. Then a well of $0.5 \mathrm{~cm}$ was made in the medium using a sterile cork hoarer; $150 \mathrm{~mL}$ of each (dist. $\mathrm{H}_{2} \mathrm{O}$ ) extracts was transferred into separate wells, after which the plates were incubated at $37^{\circ} \mathrm{C}$ for 24 to $48 \mathrm{~h}$. After incubation period, the results were observed and the diameter of incubation zone around each well was measured.

\section{Extraction of vibriocidal compound}

\section{Thin layer chromatography (TLC)}

\section{i) Preparation of thin layer plate}

The stationary phase (silica gel) was prepared as slurry with water or buffer at 1:2 ratio. It was applied to the glass plate or an inert plastic or aluminum sheet (as thin as a glass rod or pipette) using TLC applicator of $0.25 \mathrm{mM}$ thickness for analytical separation and $2.5 \mathrm{mM}$ thickness for reparation preparation.

Calcium sulphate $\left(\mathrm{CaSO}_{4}\right),{ }^{1 / 2} \mathrm{H}_{2} \mathrm{O}$ (Gypsum) (10.15\%) is incorporated to the adsorbent as a binder, since it facilitates the adhesion of the adsorbent to the plate. After the application of the adsorbent, the plates are air dried for 10 to $15 \mathrm{~min}$. This process is also known as activation of the adsorbent. The plates can be used immediately or stored in desiccators.

\section{ii) Sample preparation}

Phenols: Two grams of blue green algae (BGA) cultures were lixiviated in methanol on rotary shaker (180 thaws/mins) for $24 \mathrm{~h}$. Then the extract was filtered by using Whatman no.1 filter paper. The condensed filtrate was used for TLC (Harbone, 1998).

Amino acids: $2 \mathrm{~g}$ of BGA culture was extracted with $70 \%$ ethanol in water bath $\left(80^{\circ} \mathrm{C} / 15 \mathrm{~min}\right)$. The condensed filtrate is used for TLC.

Sterols: $2 \mathrm{~g}$ of BGA culture was extracted with $10 \mathrm{ml}$ methanol in water bath $\left(80^{\circ} \mathrm{C} / 15 \mathrm{~min}\right)$. The condensed filtrate is used for TLC (Wangner and Bladt, 1996).

Saponins: $2 \mathrm{~g}$ of BGA culture was extracted with $10 \mathrm{~mL}$ of $70 \%$ ethanol by refluxing for $10 \mathrm{~min}$. Then this extract was filtered by using What Mann no.1 filter paper. The filter is condensed, enriched with saturated n-Butanol, and thoroughly mixed. The butanol was retained, condensed and used for thin layer chromatography (Wangner and Bladt, 1996).

\section{iii) Sample application}

A line was drawn lightly with pencil, about 1.5 to $2.0 \mathrm{~cm}$ from the bottom. A scale was placed at the bottom and spotted at a distance of $1.5 \mathrm{~cm}$. The order was noted. The samples were spotted using capillary tubes at $1.5 \mathrm{~cm}$ distance between them; for preparing TLC, the sample is applied as a banal across the layer rather than as a spot.

\section{iv) Solvent preparation}

Phenols: The phenols were separated by using chloroform and methanol (27:03) solvent mixture (Harborne, 1998).

Amino acids: The amino acids were separated by using butanol (1-01), acetic acid and water (80:20:20) solvent mixture.

Sterols: The sterols were separated by using acetous, glacial acetic acid, methanol and water $(64: 34: 12: 8)$ solvent mixture (Wagner and Bladt, 1996).

Saponins: The saponins were separated by using chloroform, glacial acetic acid, methanol and water (64:34:12:8) solvent mixture (Wagner and Bladt, 1996).

\section{v) Running of sample in TLC}

The chromatographic tank is filled with developing solvent to a depth of $1.5 \mathrm{~cm}$ and equilibrated for about $5 \mathrm{~h}$. The thin layer plate is placed gently in the tank and allowed to stand for about $60 \mathrm{~min}$. It was ensured that the spots did not touch the solvent directly because capillary action can cause the solvent to display as seen in paper chromatography. And the separation of compounds takes place as the front of the solvent reaches about $1.2 \mathrm{~cm}$ from the top of the plate. The plate is removed, the front of the solvent is marked with a pencil immediately and allowed to air dry, placing the plate upside down.

\section{vi) Compounds detection}

Several methods were available to detect the separated compounds. 
Different types of spraying reagents are used to detect the different components.

Phenols: The presence of phenols in the developed chromategrams was detected by spraying folin-ciocalteu reagent. After the plates were heated at $80^{\circ} \mathrm{C}$ for $10 \mathrm{~min}$, there was a positive reaction to formation of blue color spot.

Amino acids: The presence of amino acids in the developed chromatograms was detected by spraying the $0.1 \%$ ninhydrin in acetone. After the plates were heated at $80^{\circ} \mathrm{C}$ for $10 \mathrm{~min}$, there was a positive reaction to formation of pink (or) purple spots.

Sterols: The presence of sterols in the developed chromatogram was detected by spraying folin-ciocalteu reagent. After the plates were heated at $80^{\circ} \mathrm{C}$ for $10 \mathrm{~min}$, a positive reaction to formation of blue colour spot was seen.

Saponins: The presence of saponins in the developed chromategram was detected by iodine vapors, and possible reaction to formation of yellow colour spot was seen.

\section{vii) Determination of RF value}

The $R_{f}$ values of the various bioactive compounds were calculated using the following formula. After the incubation period, the results were observed and the diameter of the inhibition zone was measured around the isolates:

\section{$\mathrm{R}_{\mathrm{f}}=$ Distance travelled by solute (measured to centre of the spot) \\ Distance travelled by solvent}

\section{Screening of antivibrio activity with the bioactive compound}

The positive bioactive compound constituents alone undergo antivibrio activity. $20 \mathrm{~mL}$ sterilized nutrient agar medium was poured into each sterile petriplate and allowed to solidify. The tested vibrio cultures were evenly spread over the appropriate media by using a sterile cotton swab. Then a well of $0.5 \mathrm{~cm}$ was made in the medium using a sterile cork hoarer; $200 \mu \mathrm{L}$ of separated constituents of phenol, amino acids and sterols were transferred into separate wells. After these plates were incubated at $37^{\circ} \mathrm{C}$ for 24 to $48 \mathrm{~h}$, the results were observed and the diameter of incubation zone around each well was measured.

\section{Screening of the various functional groups by using FTIR}

Molecular bonds vibrate at various frequencies depending on the frequencies at which they can vibrate. Based on quantum mechanics, these frequencies correspond to the ground state (lowest frequency) of several excited states (higher frequencies). One way to cause the frequency of molecular vibration to increase is to excite the bond by making it to absorb light energy. For any given transition between two states, the light energy (determined by the wavelength) must exactly equal the difference in the energy between the two states (usually ground state $\left(E_{0}\right)$ and the first excited state $\left.\left(E_{1}\right)\right)$. The energy corresponding to this transition between molecular vibrational states is generally 1 to $10 \mathrm{kcal} / \mathrm{mole}$, which corresponds to the elements and type of bonds. For any given bond, there are several specific types.

Difference in Energy states = Energy of light absorbed

$E_{0}-E_{1}=h c / l$
Where, $\mathrm{h}=$ planks constant, $\mathrm{c}=$ speed of light, and $1=$ the wavelength of light.

\section{RESULTS}

The in vitro antivibrial activities of aqueous extracts and different solvent extracts of Oscillatoria sp. and Lyngbya $\mathrm{sp}$. were evaluated by the agar-well diffusion methods against the three pathogenic Vibrio spp., Vibrio cholerae, Vibrio alginolyticus and Vibrio fluvialis. The best results of the marine cyanobacterial bioactive compounds were separated by thin layer chromatography (TLC) and their functional groups were analyzed by FTIR methods. The bioactive compounds separated through TLC were carried out for further antivibrio activity.

\section{Antivibrio activity of Oscillatoria sp. and Lyngbya}

The pyridine extract of Oscillatoria sp. and lyngbya sp. exhibited maximum zone of inhibition against Vibrio alginolyticus (20 and $19 \mathrm{~mm}$ ); and the ethyl acetate extract of Oscillatoria sp. and lyngbya sp. showed minimum zone of inhibition against Vibrio alginolyticus $(8 \mathrm{~mm})$ and no zone of inhibition against Vibrio fluvialis. Aqueous extract could not inhibit any Vibrio activity (Table 1, Figures 2 and 3 ).

\section{Bioactive compound analysis of Oscillatoria sp.}

The best results of the marine cyanobacterial (Oscillatoria sp.) bioactive compounds were separated by TLC. Bioactive compounds of Oscillatoria $s p$. revealed the presence of phenol, amino acids and sterols. The isolated bioactive compounds indicated antivibrio activity. Bioactive compounds were recorded at the $R_{f}$ values of 0.49, 0.56 and 0.57 (Table 2 and Figure 4).

\section{Antivibrio activity of bioactive compounds}

Phenolic compounds extract showed maximum zone of inhibition against Vibrio alginolyticus, Vibrio fluvialis and minimum zone of inhibition against Vibrio cholerae. Amino acids extract showed minimum zone of inhibition against Vibrio cholerae $(7 \mathrm{~mm})$ and no zone of inhibition against Vibrio alginolyticus and Vibrio fluvialis. The bioactive compounds sterols showed minimum zone of inhibition against Vibrio cholerae $(5 \mathrm{~mm})$ and no zone of inhibition against Vibrio alginolyticus and Vibrio fluvialis (Table 3 and Figure 5).

\section{Identification of functional groups by using FTIR}

FTIR spectrum of Oscillatoria sp. showed strong bands at 

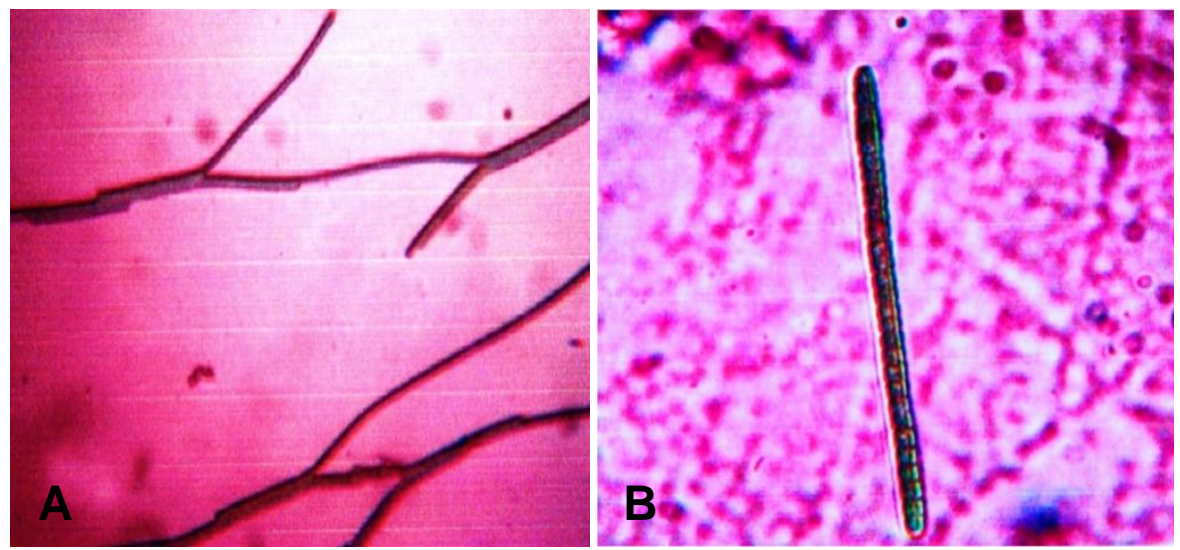

Figure 1. Photographs showing microscopic image of Oscillatoria sp. and Lyngbya sp.

Table 1. Antivibrio activity of marine cyanobacteria on Oscillatoria sp. and Lyngbya sp.

\begin{tabular}{llccc}
\hline \multirow{2}{*}{ Organism } & \multirow{2}{*}{ Pathogen } & \multicolumn{3}{c}{ Zone of Inhibition (mm) } \\
\cline { 3 - 5 } & Vibrio fluvialis & 16 & 12.5 & - \\
Oscillatoria sp. & Vibrio alginolyticus & 20 & 8 & - \\
& Vibrio cholera & 17.5 & 14 & - \\
& & & & - \\
& Vibrio fluvialis & 18 & - & - \\
Lyngbya sp. & Vibrio alginolyticus & 19 & 15 & - \\
& Vibrio cholera & 9 & 6 & \\
\hline
\end{tabular}

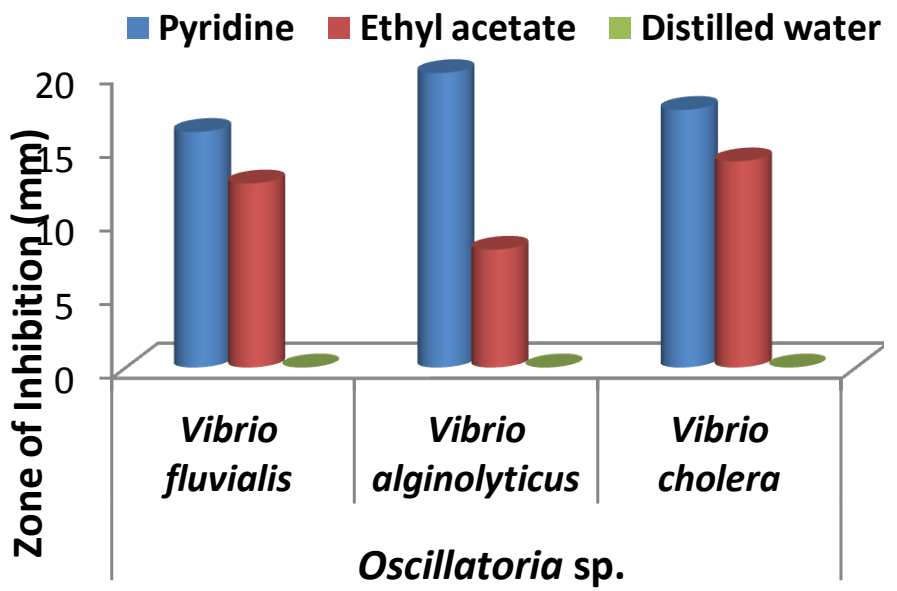

Figure 2. Zone of inhibition of antivibrio activity on Oscillatoria sp. in different extracts.

3434.89, 2832.35, 2732.66, 2160.69, 1597.45 and $136.67 \mathrm{~cm}^{-1}$. The band at $3834.89 \mathrm{~cm}^{-1}$ showed $\mathrm{N}-\mathrm{H}$ stretching vibration. The position of 2832.35 and 2723.66 $\mathrm{cm}^{-1}$ showed $\mathrm{C}-\mathrm{H}$ stretching vibration, two band and $2160.69 \mathrm{~cm}^{-1}$ peak showed $\mathrm{N}=\mathrm{N}$ stretching vibration. FTIR spectra showed that $1597.45 \mathrm{~cm}^{-1}$ peak is identified as primary amide and $\mathrm{N}-\mathrm{H}$ stretch vibration in the amide

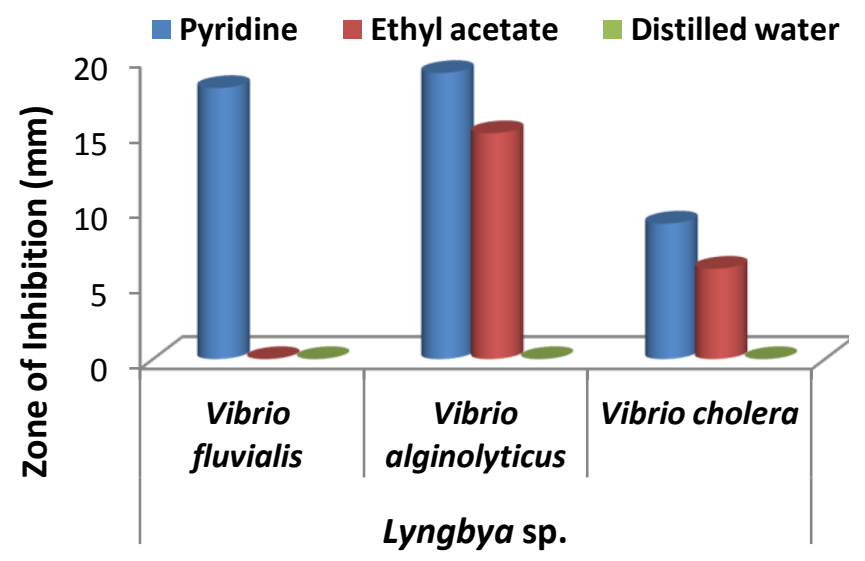

Figure 3. Zone of inhibition of antivibrio activity on Lyngbya sp. in different extracts.

Table 2. Bioactive compound analysis from Oscillatoria sp.

\begin{tabular}{lll}
\hline Bioactive compound & Result & $\mathbf{R}_{\mathbf{f}}$ value \\
\hline Amino acid & + & 0.49 \\
Phenol & + & 0.56 \\
Sterol & + & 0.57 \\
Saponins & - & - \\
\hline
\end{tabular}




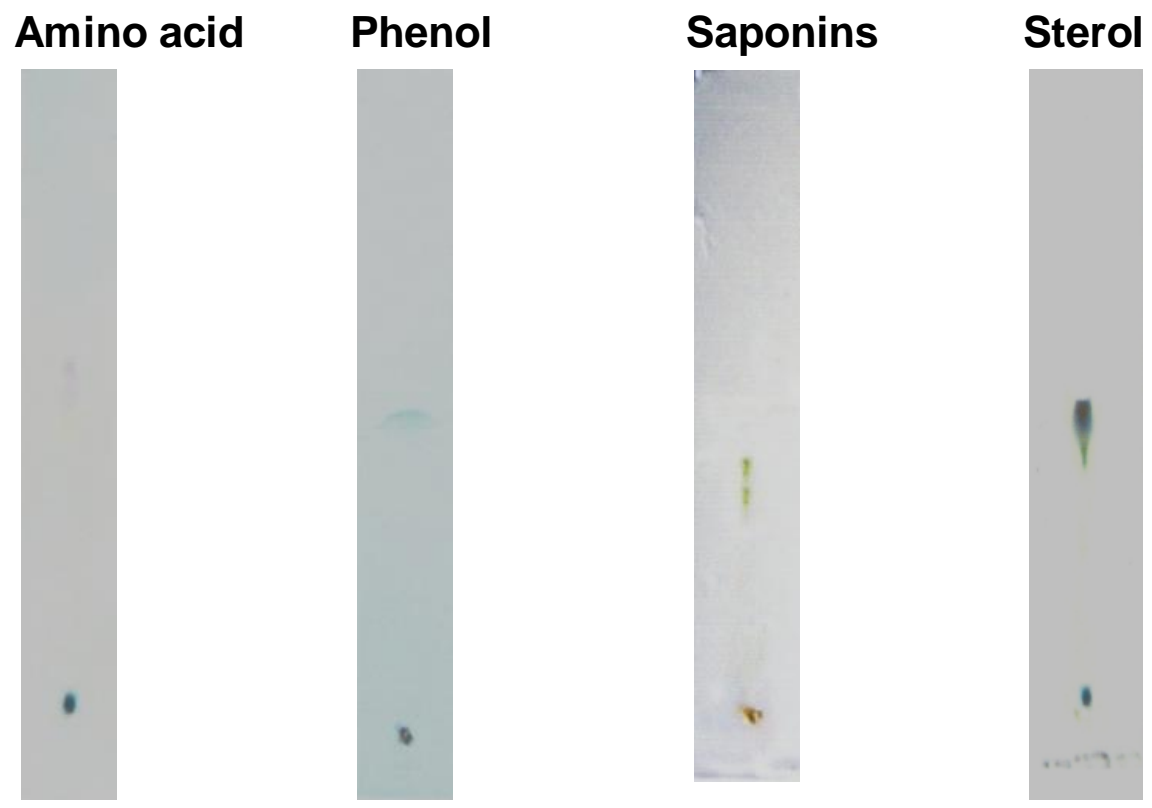

Figure 4. Thin layer chromatography (TLC) analysis of bioactive compounds from Oscillatoria sp.

Table 3. Antivibrio activity of Bioactive Compounds separated from the Oscillatoria sp. in different extracts.

\begin{tabular}{llccc}
\hline \multirow{2}{*}{ Culture } & \multirow{2}{*}{ Pathogen } & \multicolumn{3}{c}{ Zone of inhibition in mm (Bioactive compounds) } \\
\cline { 3 - 5 } & Phenol & Amino acid & Sterol \\
\hline \multirow{2}{*}{ Oscillatoria sp. } & Vibrio fluvialis & 10 & - & - \\
& Vibrio alginolyticus & 13 & - & - \\
& Vibrio cholerae & 8 & 7 & 5 \\
\hline
\end{tabular}

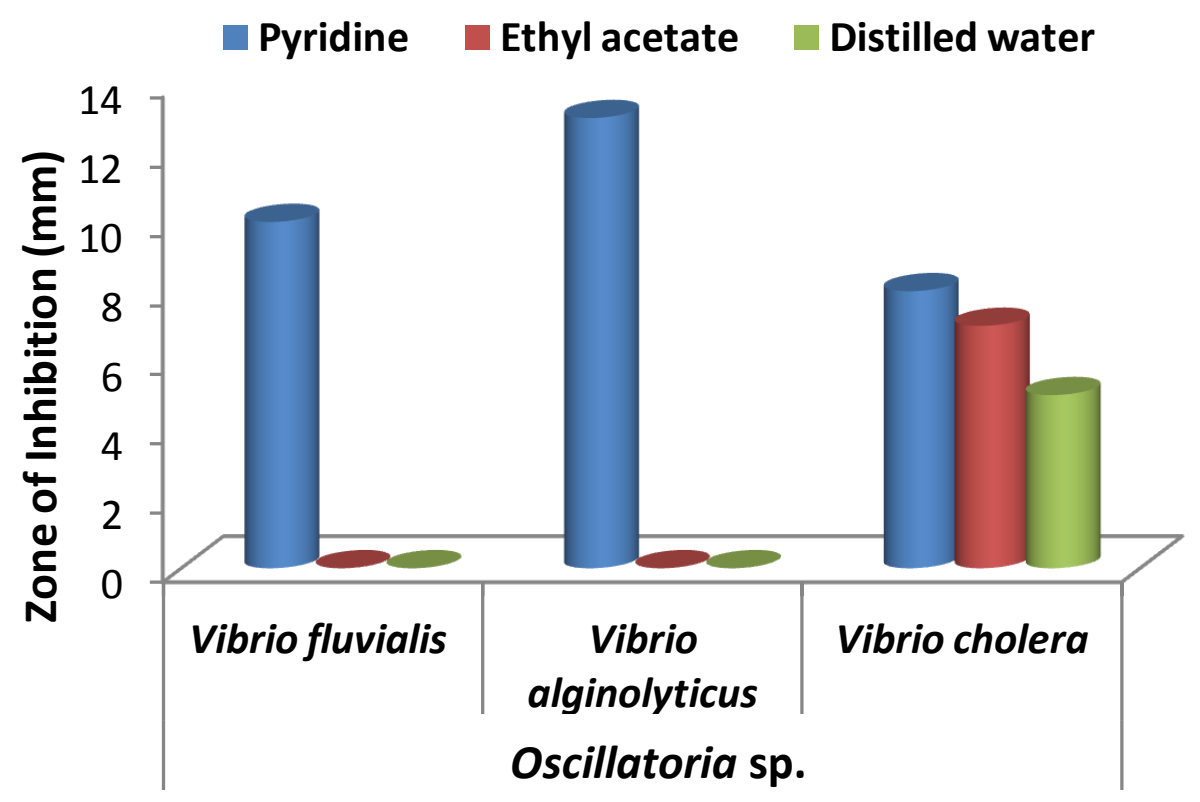

Figure 5. Zone of inhibition of antivibrio activity on bioactive compounds separated from Oscillatoria sp. 


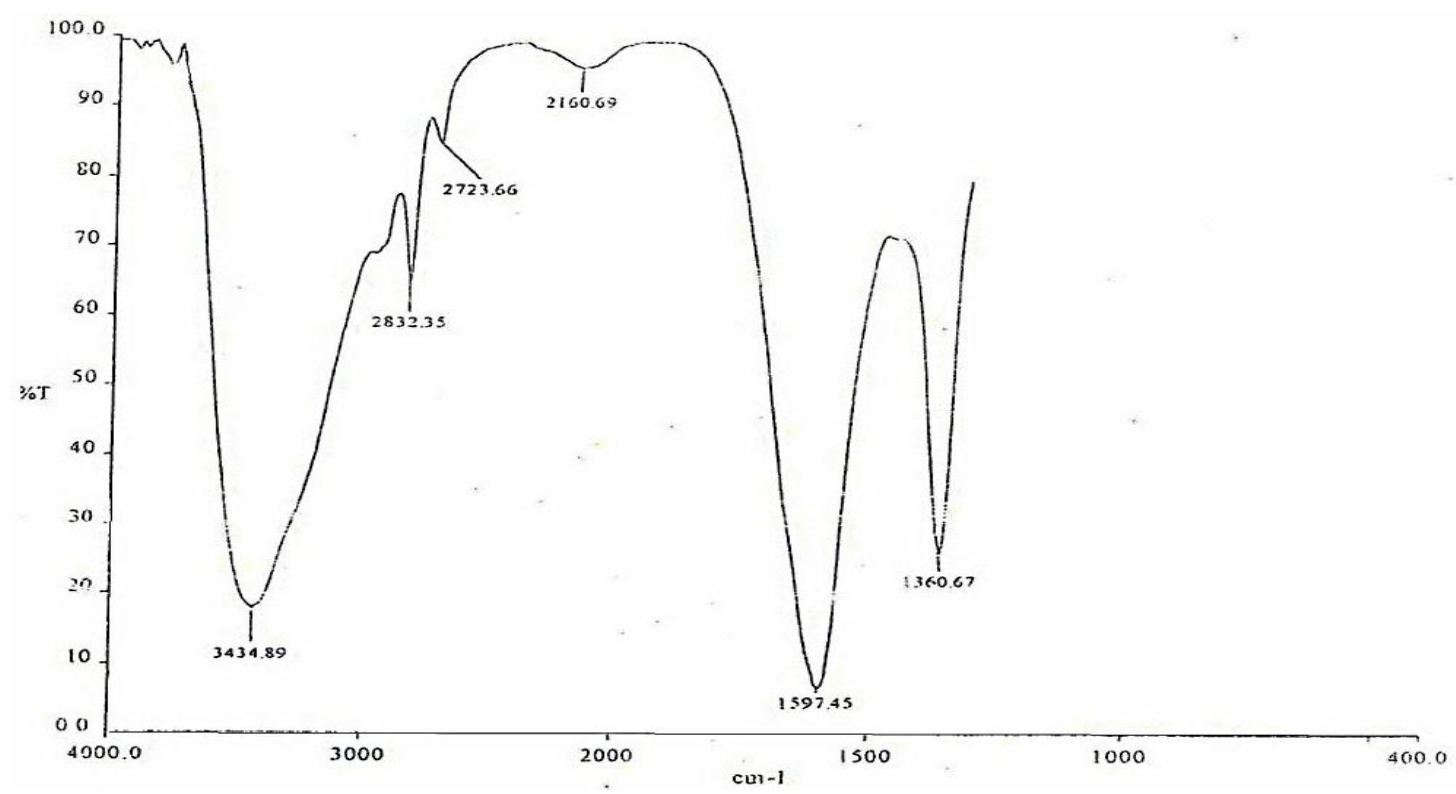

Figure 6. Fourier Transform Infrared (FT-IR) spectroscopy of isolated bioactive compounds from Oscillatoria sp.

linkage of protein shown in Figure 6.

\section{DISCUSSION}

Various strains of marine cyanobacteria are known to produce intracellular and extracellular metabolites (Noaman et al., 2004), with diverse biological activities such as antibacterial, antifungal and antiviral activities. In the present study, efforts made to identify antivibrio activity agent in cyanobacteria have revealed several promising lead compounds. Most studies have only been done on in vitro assays, but a few studies have been done to screen cyanobacteria for production of antivibrio species substance.

In this study, antivibrio activity compound and phytochemicals were screened from cyanobacteria using thin layer chromatography methods. The cured extract of Oscillatoria sp. exhibited maximum zone of inhibition against Vibrio species and the crude extracts were further separated by thin layer chromatography. The separated fraction was evaluated for vibriocidal activity. In general, isolation of bioactive compounds from marine cyanobacteria is done with two objectives: one is to discover new compounds for pharmaceutical, agricultural or bio control applications; another one is to better understand individual organisms within their natural communities (Kulik et al., 1995; Febles et al., 1995). Moreover, many bioactive compounds may be extracted into the environment due to the stress of survived cyanobacteria (Nicholson et al., 2000; Soltani et al., 2006). The activated fraction was further analyzed by Fourier transforms infrared spectroscopy (FTIR) and the fraction shows the presence of phenols, aminoacids, sterols and saponins.

\section{Conclusion}

In the present study it is concluded that the Oscillatoria sp.and Lyngbya sp. producing antivibrio agent reduced zone of inhibition against Vibrio alginolyticus. Vibrio Cholerae and Vibrio fluvialis. Between both species of marine cyanobacteria, Oscillatoria sp. alone exhibited maximum inhibition against three different Vibrio spp. As a result, the Oscillatoria sp. was analyzed by FTIR; phenol compound was observed due to the presence of alcohol $(-\mathrm{OH})$ group in spectrum. The study has suggested that, antivibrio metabolites of marine cyanobacteria are of special interest in the development of new harmless environment.

\section{ACKNOWLEDGEMENT}

The authors acknowledge the Department of Microbiology, Urumu Dhanalakshmi College, Tiruchirappalli620019. Tamil Nadu, India for providing lab and Internet facilities.

\section{REFERENCES}

Desikachary TV (1959). Cyanophyta, ICAR Monographs on Algae.ICAR New Delhi p. 686.

Febles $\mathrm{Cl}$, Arias A et al (1995). In vitro study of antimicrobial activity in algae (Chlorophyta, Phaeophyta and Rhodophyta) collected from the coast eg. Tenerite (In Spanish), Aunario del Estudios Canarios. 34:181-192.

G'omez-Gil B, A Roque, Turnbull JF (2000). The use and selection of probiotic bacteria for use in the culture of larval aquatic organisms. Aquaculture 191:259-270.

Ghasemi Y, Tabatabaci Yazdi M, Shokravi S, Soltani N, Zarrini G (2003). Antifungal and antibacterial activity of paddy fields marine cyanobacteria from the north, Iran J Sci., 14(3): 203-209. 
Harborne JB (1998). In Phytochemical Methods; A Guide to Modern Techniques of Plant Analysis, Chapman and Hall, London, United Kingdom. 40-106.

Kulik MM (1995). The potential for using marine cyanobacteria (blue green algae) and algae in the biological control of plant pathogenic bacteria and fungi, Eur. J. Plant Pathol. 101: 585-599.

Marshall JA, M de Salas, T Oda, G Hallegraeff (2005). Superoxide production by marine microalgae: I. Survey of 37 species from 6 classes. Mar. Biol. 147:533-540.

Michael Hogan C (2010). Bacteria. Encyclopedia of Earth. eds. Sidney Draggan and C.J.Clevel and National Council for Science and the Environment, Washington DC.

Mundt S, Kreitlow S, Nowotny A, Effmert U (2001).Biological and pharmacological investigation of selected cyanobacteria. Int. J. Hyg. Environ. Health. 203: 327-334.

Nicholson WL, Munakata N, Horneck G, Melosh HJ, Setloe P. (2000).Resistance of Bacillus endospores to extreme terrestrial and extraterrestrial environments. Microbiol. Mol. Rev. 64(3): 548-572.

Noaman NH, Fattah A, Khaloafa M, Zaky SH (2004).Factors affecting antimicrobial activity of Synechococcusleopoliensis. Microbiol. Res. 159: 395-402.

Perez C, Pauli M, Bazerque P (1990). An antibiotic assay by agar-well diffusion method. Acta Biologiaeet Med. Exper. 15: 113-115.

Rania MAA, Hala MT (2008).Antibacterial and antifungal activity of marine cyanobacteria and green microalgae, Evaluation of medium components by placket Burman design for antimicrobial activity of Spirulina platensis. Glob. J. Biotechnol. Biochem. 3: 22-31.
Soltani N, Khavari-Nejad RA, TabatabaeiYazdi M, Shokravi SH, Fernandez- Valiente E (2006). Variation of nitrogenase activity, photosynthesis and pigment of the cyanobacterium Fischerella ambigua strain FS18 under different irradiance and $\mathrm{pH}$ values. World J. Microbiol. Biotechnol. 22: 571-576.

Verschuere L, Rombaut G, Sorgeloos P, Verstraete W (2000). Probiotic bacteria as biological control agents in aquaculture. Microbiol. Mol. Biol. Rev. 64:655-671.

Vine NG (2004).Towards the development of a protocol for the selection of probiotics in Marine Fish Larvicuture.PhD Thesis. Rhodes University, Grahamstown, South Africa.

Wagner H, Bladt S (1996).Plant drug analysis, A thin-layer chromatography atlas, 2nd ed. Berlin, Springer. pp.306-364. 